\title{
Primary Cilia in the Breast and Breast Cancer
}

\author{
Kun Yuan ${ }^{1}$, Rosa Serra ${ }^{2,3}$, Andra R. Frost ${ }^{*}, 1,2,3$ \\ Departments of ${ }^{1}$ Pathology and ${ }^{2}$ Cell Biology and the ${ }^{3}$ Comprehensive Cancer Center, University of Alabama at \\ Birmingham, Birmingham, AL 35294, USA
}

\begin{abstract}
Primary cilia are non-motile, microtubule-based appendages extending from the surfaces of most vertebrate cells. The function of primary cilia is cell-type dependent. However, they are known to serve as sensors of the extracellular environment by sensing mechanical forces and chemical constituents in several different cell types. They also function as critical platforms for several signaling pathways, including hedgehog and platelet derived growth factor (PDGF) signaling, and function in maintaining cell polarity. While present on many types of normal cells, including epithelial cells of the breast, they have been shown to be decreased or absent in many cancers. This review discusses the relative decrease in primary cilia in a variety of cancers and the known consequences of the presence or absence of primary cilia during tumorigenesis. This review also addresses possible etiologies for the loss of primary cilia in cancer, potential functions for primary cilia in human breast epithelium and subsequent consequences of the loss of primary cilia during breast carcinogenesis.
\end{abstract}

Keywords: Primary cilia, breast, cancer.

\section{INTRODUCTION}

Primary cilia are non-motile, microtubule-based appendages extending from the surfaces of most vertebrate cells. Primary cilia arise from a basal body, derived from the mother centriole, and the ciliary axoneme which is covered by a specialized plasma membrane. The process of intraflagellar transport (IFT) is responsible for building and maintaining the structure and function of primary cilia by transporting structural components up and down the microtubular structure $[1,2]$. Disruption of a component of the Kinesin-II motor complex, Kif3a, disables anterograde IFT and leads to the failure of ciliogenesis [3]. Likewise, the absence of a core component of the IFT particle, IFT88/Tg737/Polaris, results in the loss of primary cilia [46]. Defects in the structure, assembly or function of primary cilia are known to play a role in the development of several congenital diseases, including polycystic kidney disease [7]. The function of primary cilia is believed to be cell-type dependent; however, they have been shown to regulate cell polarity, serve as mechanosensors responsible for cellmicroenvironment interactions, and they serve as key participants in several signaling pathways, including those for hedgehog and platelet derived growth factor (PDGF) [7, $8]$. The presence of primary cilia is also closely linked to the cell cycle, with primary cilia typically present in quiescent cells in the $\mathrm{G}_{0}$ phase of the cell cycle [9]. Consequently, primary cilia may participate in regulating the cell cycle. Although primary cilia have been described on myoepithelial and luminal epithelial cells in the human breast by electron microscopy $[10,11]$, the functional significance of IFT and primary cilia in the breast is unknown. Many of the

*Address correspondence to this author at the Departments of Pathology, University of Alabama at Birmingham, 640B Kaul Human Genetics Building, $70120^{\text {th }}$ Street South, Birmingham, AL 35294, USA; Tel: 205934-2746; E-mail: afrost@uab.edu processes known to be regulated by primary cilia, such as cell-microenvironmental interactions, hedgehog and PDGF signaling, and cell polarity, are also important in cancer development and progression, raising the possibility that primary cilia may participate in the development and progression of cancer, including breast cancer.

\section{PRIMARY CILIA ARE DECREASED OR ABSENT IN CANCER}

Studies to assess the presence of primary cilia in cancer are limited, but most have reported a relative decrease in the incidence of primary cilia in multiple types of cancer in comparison to their normal cellular counterparts. While primary cilia were present on most $(87 \%)$ epithelial cells of the normal renal nephron, their incidence in the epithelial cells comprising renal cell carcinomas varied from 0 to $83 \%$ with most carcinomas having an incidence of primary cilia of less than $10 \%$ [12]. In this analysis, two different histologic types of renal cell carcinomas were analyzed, clear cell carcinoma and papillary carcinoma. The rate of ciliation was significantly higher in the papillary carcinomas than the clear cell carcinomas. This difference was associated with inactivation of the von Hippel-Lindau tumor suppressor gene, which functions in the maintenance of primary cilia [12]. In basal cell carcinomas of the skin, some were extensively ciliated $(5 / 8)$, similar to normal keratinocytes, whereas the remainder of basal cell carcinomas exhibited no primary cilia (3/8) [13], indicating a loss of primary cilia in a subset of these cancers. In medulloblastomas, 39\% (15/38) were ciliated, whereas the remainder was not. The presence of primary cilia was more frequent in the desmoplastic histologic subtype of medulloblastoma (present in 5 of 6), whereas, the absence of primary cilia was associated with the anaplastic histologic subtype (present only in 1 of 9) [14]. Individuals with anaplastic medulloblastomas have a particularly poor prognosis [15]. Whether the absence of primary cilia contributes to this poor prognosis remains to be 
determined. Primary cilia were reported to be absent in the malignant epithelial cells of human pancreatic carcinomas, as well as the putative in situ precursor lesions, pancreatic intraepithelial neoplasia [16]. However, primary cilia were identified in two pancreatic cancer cell lines, CFPAC-1 and PANC-1, when grown in culture [17]. Although primary cilia were shown to be frequently expressed in a normal human astrocyte cell line, in 5 astrocytoma/glioblastoma cell lines, primary cilia were either greatly reduced in number, entirely absent or had an aberrant structure [18]. Similarly in human breast cancer tissues, we have found that primary cilia are extremely rare or entirely absent in cancer epithelium compared to histologically normal breast epithelium, and are absent or markedly decreased in breast cancer cell lines in comparison to epithelial cell lines derived from histologically normal breast [65].

\section{POTENTIAL MECHANISMS UNDERLYING THE LOSS OF PRIMARY CILIA IN BREAST CANCER}

Cancers are characterized by multiple chromosomal mutations, including chromosomal loss or gain, and resulting aberrant protein expression. Therefore, it is possible that the decrease or loss of primary cilia is a result of mutations in genes known to be important for the assembly or maintenance of primary cilia. The work of multiple investigators has resulted in a collection of known ciliaassociated genes or gene products [19], which provides a pool of gene candidates that may be altered in cancer and contribute to the decreased occurrence of primary cilia. Furthermore, attempts to define the genomic landscape in breast cancer have included sequencing the genomes of several breast cancers $[20,21]$. It was shown that several cilia-associated genes, including Gli1, RPGRIP1, and DNAH9, are commonly mutated in breast cancer. RPGRIP1 is located in the basal body and is a GTPase regulator [22]. DNAH9 is a component of the dynein motor, which is important for IFT [23]. Gli1 is a component of the hedgehog signaling pathway, which is discussed in detail below. The direct effects of mutations in RPGRIP1, DNAH9 or Gli1 on breast cancer progression are not known.

It has also been shown that some of the proteins that regulate the assembly or disassembly of primary cilia also regulate the cell cycle so alterations in the expression or function of proteins that regulate the cell cycle could underlie the loss of primary cilia in some cancers. Primary cilia arise from the mother centriole in cells that are not actively dividing [24]. Centrosomes are composed of a pair of centrioles which are surrounded by pericentriolar material [25]. Centrosomes are essential for cell division as they are responsible for organization of the mitotic spindle and are important for cytokinesis. Therefore, the centrioles of cells that are engaged in mitosis are not available for ciliogenesis. This is why primary cilia are commonly found in quiescent, non-proliferating cells. Many of the proteins that regulate centrosomal function and cell cycle progression also regulate primary cilia formation. For example, the centrosomal protein CP110 is phosphorylated by cyclin dependent kinase 2 (CDK2) during cell cycle progression and is recruited to the centrosome by Cep97, another centrosomal protein. Depletion of either CP110 or Cep97 increases the proportion of cells that display primary cilia, indicating that these proteins normally function to inhibit primary cilia formation during cell proliferation [26]. The ability of CP110 to inhibit the formation of primary cilia requires its interaction with Cep290, another centrosomal protein that functions to promote ciliogenesis through cooperation with Rab8a [27]. Therefore, loss of Cep290 or enhanced expression of CP110 in cancer cells may decrease their ability to assemble primary cilia.

Because of the close association between ciliogenesis and proliferation, it has been theorized that the decrease in primary cilia in cancer is a result of a high rate of proliferation in cancer. This theory has been addressed in several studies, which have concluded that the lower incidence of primary cilia in cancer is not simply a result of a higher rate of proliferation $[12,17,28]$. In renal cell carcinomas, there was no association between the rate of proliferation, as determined by immunohistochemical staining for Ki-67, and the incidence of primary cilia [12]. Similarly, the majority of epithelial cells in pancreatic carcinomas were not proliferating, as determined by Ki-67 and PCNA labeling, and yet all were devoid of primary cilia. Therefore, it was concluded that ongoing proliferation was not responsible for the absence of primary cilia [16]. No primary cilia were identified in three human prostate cancer cell lines, PC-3, LNCaP, and 22RV1 cells, even in proliferation-inhibiting culture conditions (i.e., confluent growth and low serum), leading to the conclusion that the absence of primary cilia was not a result of proliferative activity [28].

A decrease or dysfunction of proteins responsible for the assembly of primary cilia may occur in cancer. Alternatively, the disassembly of primary cilia may be accelerated in cancer. For example, the prometastatic scaffolding protein HEF1 and the oncogenic Aurora A kinase (AuroraA) localize to the basal body of primary cilia. HEF1 stabilizes and activates AuroraA. Activated AuroraA phosphorylates HDAC6, an $\alpha$-tubulin deacetylase that promotes microtubule destabilization, and consequently induces ciliary disassembly [29]. Therefore, increased expression or activation of AuroraA or HDAC6 in cancer may lead to a decrease in primary cilia.

\section{POSSIBLE CONSEQUENCES OF DECREASED PRIMARY CILIA IN BREAST CANCER}

Primary cilia have been documented to perform multiple functions in a cell type dependent fashion, including mediating hedgehog and PDGF signaling, mechanosensation and cell polarity. The loss of primary cilia in breast cancer may result in alterations in these diverse biological processes and contribute to cancer development and progression. The functional consequences of the loss of primary cilia in cancer are just beginning to be investigated. To date, only two studies address this issue directly and only in the context of hedgehog signaling.

\section{Primary Cilia in Hedgehog Signaling}

Primary cilia are required for normal hedgehog signaling. Upon initiation of signaling by Hedgehog ligand, the pathway receptor Patched 1 (Ptch1) releases its suppression of another transmembrane protein, Smoothened (Smo), thereby activating hedgehog signaling [30, 31]. With pathway activation, Ptch1 exits the primary cilium, whereas 
Smo enters the primary cilium $[32,33]$. Localization of Smo to primary cilia appears to be essential for its activation. Hedgehog dependent transcription is mediated by three transcription factors, Gli1, Gli2 and Gli3. Data generated primarily in murine model systems indicates that Gli1 is exclusively a transcriptional activator, whereas Gli2 is predominantly a transcriptional activator and Gli3 is primarily a transcriptional repressor [34, 35]. Hedgehog stimulated activation of Gli2 and the processing of Gli3 to its repressor form is dependent upon their localization to primary cilia [36].

A context-dependant dual role for primary cilia, as either a tumor suppressor or as a promoter for cancer development, has been demonstrated in two types of cancers known to be driven by hedgehog signaling, basal cell carcinomas of the skin and medulloblastomas $[13,14]$. In two mouse models of basal cell carcinoma of the skin, one driven by a constitutively active Smo and a second driven by activated Gli2, the presence of primary cilia in the skin was ablated by conditional deletion of Kif3a. In those basal cell carcinomas driven by Smo, ciliary ablation inhibited tumor development; whereas, in basal cell carcinomas driven by activated Gli2, the absence of primary cilia accelerated tumor development [13]. These findings suggest that the presence of primary cilia is essential for the ability of Smo to mediate hedgehog signaling and induce carcinogenesis, whereas, stimulation of hedgehog signaling by activated Gli2 does not require primary cilia, and the presence of primary cilia may inhibit carcinogenesis induced by activated Gli2, perhaps by sequestration of Gli2 in cilia. Similarly, in two mouse models of medulloblastoma, one driven by a constitutively active Smo and one driven by activated Gli2, primary cilia were ablated in the cerebellar granule neuron precursors by removal of Kif3a or IFT88 [14]. In the Smo-driven model, depletion of primary cilia resulted in inhibition of proliferation of granule neuronal precursors and subsequent tumorigenesis. It was already known that proliferation of these cells was dependent on Hedgehog ligand and, as expected, loss of cilia blocked normal hedgehog signaling in these cells. Activated Gli2 alone was not sufficient to induce medulloblastoma in mice, whereas mice with depletion of primary cilia demonstrated tumors in the presence of activated Gli2 [14]. It was also shown that primary cilia were frequent in a subset of human medulloblastomas with activation of hedgehog or wnt signaling, whereas primary cilia were absent in those without activation of these pathways [14]. These data in basal cell carcinoma and medulloblastoma support a dual role for primary cilia as activators and suppressors of tumorigenesis, depending on the molecular mechanisms responsible for the initial formation of the tumor. In cancers driven by activation of Smo, primary cilia facilitate tumor formation, whereas, in cancers driven by hedgehog pathway members that function downstream of Smo and do not require primary cilia for their activation, such as an activated Gli2, loss of primary cilia promotes tumor formation. Data suggest that in breast cancer, at least a subset of tumors have activated hedgehog signaling, either by methylation and reduced expression of Ptch1 or increased expression of Smo or Hedgehog ligand [37-40]. If this is indeed the case, those breast cancers in which activated hedgehog signaling promotes breast cancer development or progression should contain some ciliated cancer epithelial cells. However, expression and activity of Gli1 in breast cancer may be increased independently of canonical Hedgehog signaling, via Transforming growth factor $\beta$ or possibly Ras/Erk signaling [41, 42]. Furthermore, Gli1 does not require primary cilia for activation [36]; therefore, Gli1 may contribute to the development or progression of those breast cancers that lack primary cilia.

\section{Primary Cilia in PDGF Signaling}

Primary cilia also serve as a critical platform for PDGF signaling [43]. Platelet derived growth factor receptor $\alpha$ $(\operatorname{PDGFR} \alpha)$ localizes to primary cilia in murine embryonic fibroblasts. Furthermore, activation of Akt and ERK1/2 pathways by PDGF ligand required the presence of primary cilia [43]. PDGF signaling has been shown to promote epithelial to mesenchymal transition and metastasis in animal models of breast cancer and expression of PDGFR $\alpha$ is a poor prognostic indicator in breast cancer [44, 45]. Therefore, similar to hedgehog signaling, the presence of primary cilia in cancer may be required for any promotional effect of PDGFR $\alpha$ signaling.

\section{Primary Cilia in Mechanosensation and Regulation of Cell Polarity}

The functions of primary cilia in normal breast epithelium are unknown; however, primary cilia are known to transmit both chemical (as described above) and mechanical signals. Mechanosensation and mechanotransduction are linked to cell polarity and Wnt signaling. The discovery that primary cilia function as mechanosensors coincided with the finding that mutations in IFT88 caused cyst formation in the kidney [46]. In the renal tubule, primary cilia extend from lining epithelial cells into the tubular lumen. Fluid flow through the tubule bends primary cilia, which initiates intracellular calcium signaling [47, 48]. In autosomal dominant and recessive polycystic kidney disease, mutations in several genes involved in ciliary function, specifically polycystin-1, polycystin 2 and PKHD1 polyductin/fibrocystin, result in cyst formation. In the absence of these proteins, the bending of primary cilia does not initiate intracellular calcium signaling [8, 49-51]. The studies in polycystic kidney disease indicate that mechanical signals transmitted through primary cilia are required for normal renal cell polarity, growth and differentiation.

Cell polarity and mechanotransduction are linked in the kidney though Wnt signaling. The Wnt family of proteins consists of at least 19 members whose functions contribute to the regulation of a wide range of cellular processes [52, 53]. Wnt proteins activate many signaling cascades that can be broadly divided into two general categories 1) the canonical, ß-catenin pathway and (2) the non-canonical pathways that do not involve $\beta$-catenin. The canonical pathway occurs after ligand binding when the cytoplasmic protein, dishelleved (Dsh), acts to inhibit the activity of GSK-3ß, which is in a complex with the Adenomatous Polyposis Coli protein (APC) and Axin resulting in stabilization of the $\beta$-catenin protein and its subsequent translocation to the nucleus. Nuclear $\beta$-catenin associates with the Lymphoid Enhancer Factor/T-Cell-Specific Transcription Factor (LEF/TCF) family of transcription factors and activates transcription of Wnt target genes. There are several non-canonical pathways and primary cilia are 
implicated in the regulation of the planar cell polarity pathway (PCP) [54-56]. PCP regulates cell polarity, migration, and orientation of cells during embryonic development. Recent evidence suggests that primary cilia function as a molecular switch between canonical and PCP Wnt signaling. A key ciliary protein in this switch is Inversin (Invs) [57]. Overexpression of Invs inhibits canonical Wnt signaling and knock-down of Invs results in loss of PCP signaling. Invs forms a complex with Dsh that targets Dsh for degradation thus inhibiting canonical signaling. In response to mechanical signals from fluid flow in the kidney, Invs and Dsh localize to the membrane where they can interact with other PCP signaling molecules, thus, promoting PCP signaling and proper cell polarity [57]. In the absence of cilia, PCP signaling is disrupted, canonical Wnt signaling increases, and cell polarity is disrupted while proliferation is increased. The end result is cyst formation. Therefore, primary cilia are also important for the regulation of normal cell polarity.

Additional evidence of a role for primary cilia in cell polarity or orientation comes from data suggesting that primary cilia direct the migration of smooth muscle cells. It was found that in the scratch wound assay, the location of primary cilia within the cells was aligned between the nucleus and the wound edge. In addition, smooth muscle cells with primary cilia were more migratory than those without primary cilia [58].

Primary cilia in some cell types, such as chondrocytes, have been theorized to also act as mechanosensors by extending into the extracellular matrix surrounding the cells and thus receive various mechanical singals by direct interaction with the matrix [59]. By electron microscopy, the primary cilia of chondrocytes project into the collagen fibers and condensed proteoglycans of the cartilaginous extracellular matrix. In addition, the primary cilia of chondrocytes, surrounded by extracellular matrix, exhibit a variety of bending patterns when examined by electron microscopy, as well as confocal and tomographic microscopy, suggesting deflection by the matrix [60,61]. The primary cilia are also closely associated with the Golgi apparatus and microtubule cytoskeleton, supporting the potential for outside-in signaling from the extracellular matrix through the primary cilium and into the cell [59].

Primary cilia also project from epithelial cells lining the biliary track, and the loss or dysfunction of primary cilia is implicated in cystic diseases of the liver [62]. In addition, primary cilia were found to function as sensors of osmolarity of luminal contents in the biliary tree. This function is mediated by TRPV4, a $\mathrm{Ca}(2+)$-permeable ion channel, which localizes to primary cilia. Biliary epithelial cells respond to hypotonicity of luminal contents with an increase in intracellular $\mathrm{Ca}(2+)$, a response that requires the participation of TRPV4, primary cilia, and extracellular calcium. This response induces bicarbonate secretion [63].

In histologically normal human breast, we and others have found primary cilia in occasional luminal epithelial cells lining ducts and acini and extending into the lumina (Fig. 1A) $[10,65]$. Whether the primary cilia on these lining epithelial cells also function as mechanosensors or sensors of osmolality of breast secretions remains to be determined. If the primary cilia serve as mechanosensors, their loss in these cells could interfere in mechanosensation and contribute to the cyst formation of fibrocystic disease. The loss of primary cilia in luminal cells could also interfere in normal cell polarity, and thereby contribute to cancer formation. Their

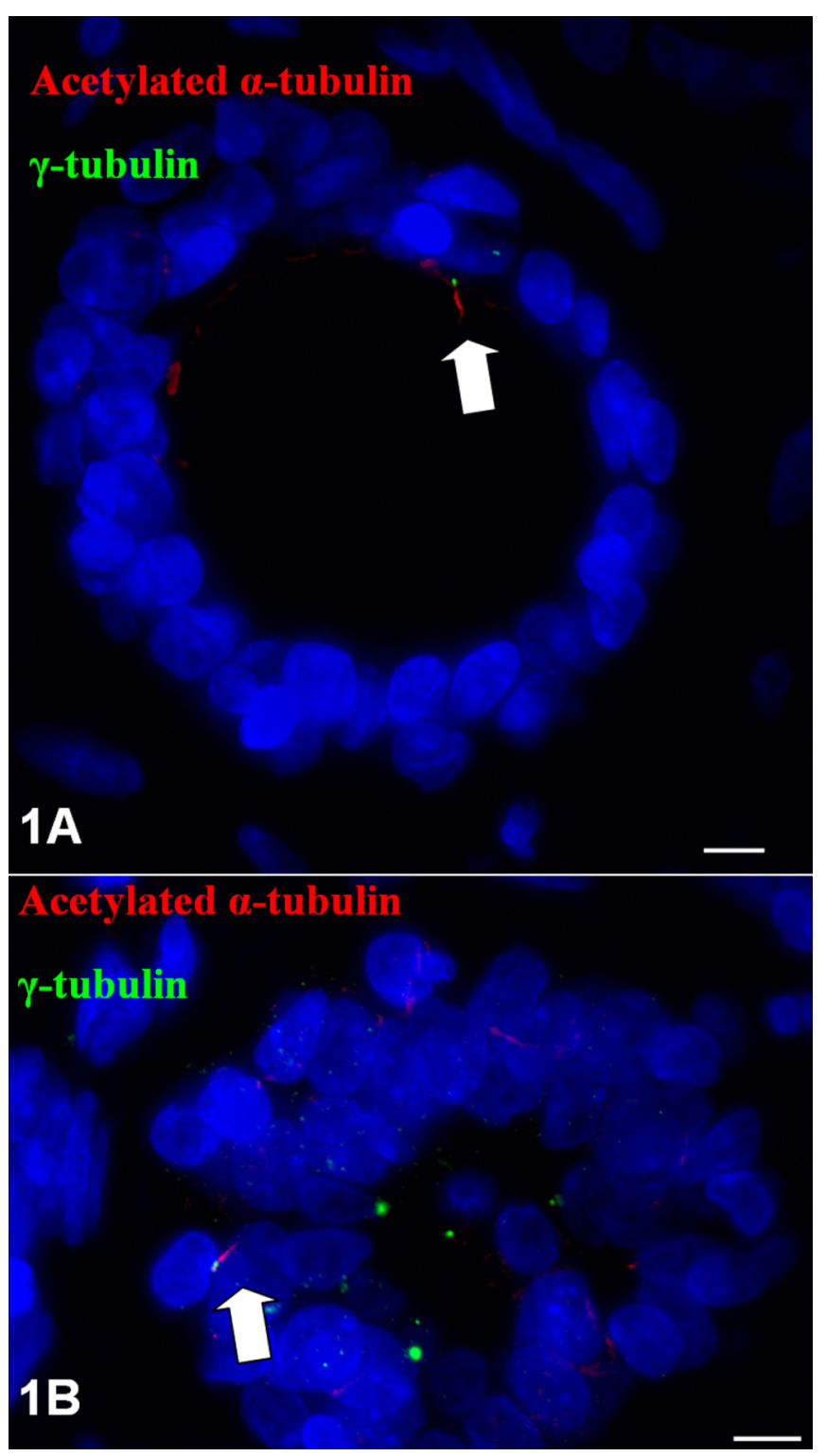

Fig. (1). Primary cilia are present in epithelial cells of the histologically normal breast. In these photomicrographs, the primary cilium is identified by co-immunofluorescence for acetylated $\alpha$-tubulin (red, axoneme), which forms the microtubule core of the primary cilium, and $\gamma$-tubulin (green), which labels the centrosome from which the primary cilium arises. The nuclei are stained with DAPI (blue). The photomicrographs are merged images with an original magnification of 1000x. The scale bar equals $10 \mu \mathrm{M}$. A) A primary cilium (arrow) is localized to a luminal epithelial cell of a small duct and extends into the lumen. B) A primary cilium (arrow) is located on a non-luminal cell, which is consistent with a myoepithelial cell, of an acinus. Coimmunofluorescence for acetylated $\alpha$-tubulin and keratin 14 (a myoepithelial cell marker) was also performed to confirm localization of primary cilia in myoepithelial cells (not shown) [65]. 


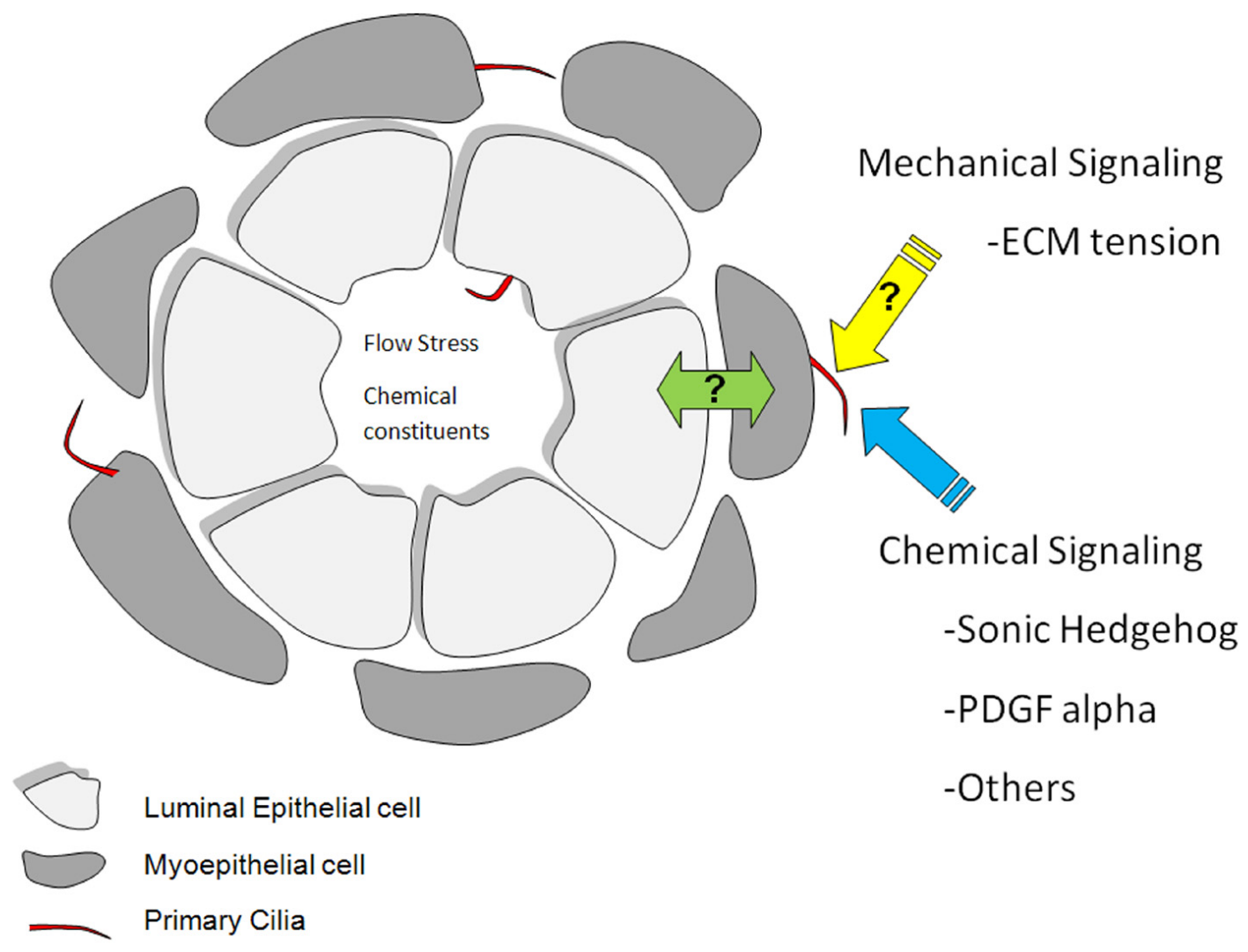

Fig. (2). A schematic representing possible functions of primary cilia located on luminal or myoepithelial cells of the breast. Primary cilia in luminal epithelial cells may function as mechano- or chemosensors of their microenvironment, in particular the luminal contents. The primary cilia located on myoepithelial cells may extend into basement membrane and sense tensional forces in basement membrane and/or extracellular matrix. Additionally, the primary cilia in either cell type may serve as platforms for hedgehog, PDGF or other signaling pathways with ligands located extracellularly and "sensed" by the primary cilia. These potential functions of primary cilium in breast epithelium may serve to mediate communication between the extracellular matrix and epithelial cells or between luminal epithelial cells and myoepithelial cells. Loss of primary cilia would interfere in any of these processes and could contribute to cancer development.

presence or absence in cancer epithelial cells may also impact cancer cell migration. We have also found that primary cilia are more frequent on myoepithelial cells than luminal epithelial cells of ducts and lobules (Fig. 1B) [65]. The primary cilia on myoepithelial cells may function as mechanosensors and sense stress in the basement membrane and extracellular matrix. Such a sensory function for primary cilia on myoepithelial cells may be important for regulation of duct or acinar structure. If so, loss of primary cilia on these cells during carcinogenesis may interfere in the crosstalk between myoepithelial cells and extracellular matrix or luminal epithelial cells, which in turn may contribute to abnormalities in epithelial structure or the sizes of ducts or acini (Fig. 2).

\section{Primary Cilia and the Cell Cycle}

While the close relationship between the cell cycle and the presence of primary cilia is well known, a role for primary cilia in regulating the cell cycle is not established. However, it has been shown that IFT88, which is required for primary cilia formation, associates with the centrosome [64]. Depletion of IFT88 in non-ciliated Hela cells promotes cell cycle progression and cell proliferation, while overexpression of IFT88 prevents G1-S transition and induces apoptosis. The cell-cycle regulating function of IFT88 may arise from its association with Che-1 protein, which in turn interacts with $\mathrm{Rb}$ to modulate G1-S transition [64]. Whether the absence of primary cilia directly promotes cell cycle progression is a matter of ongoing study. If this proves to be true, then the loss of primary cilia during carcinogenesis would have obvious promotional effects on cancer growth.

\section{SUMMARY AND FUTURE DIRECTIONS}

Much remains to be learned about the function of primary cilia in the normal breast and how the loss of primary cilia in the breast may impact cancer development or progression. However, studies to date in basal cell carcinomas and medulloblastomas document that primary cilia are important determinants of carcinogenesis. It is possible to modulate the presence of primary cilia by silencing or expressing proteins known to regulate or contribute to the assembly or disassembly of primary cilia. Therefore, it may be possible to develop pharmacologic agents that can inhibit or promote the presence of primary cilia. Determining the feasibility of targeting primary cilia in a clinical setting will require elucidating the physiological functions of primary cilia in the normal breast and the pathologic phenotypes that result from altering the presence of the primary cilia through in vivo and in vitro experimental approaches. 


\section{ACKNOWLEDGEMENTS}

This work was supported by the Department of Defense Breast Cancer Research Program (BC083907), the American Cancer Society (RSG-05-207-01-TBE), and the Susan G. Komen Foundation (BCTR0707453).

\section{REFERENCES}

[1] Pan J, Wang Q, Snell WJ. Cilium-generated signaling and ciliarelated disorders. Lab Invest 2005; 85: 452-63.

[2] Davenport JR, Yoder BK. An incredible decade for the primary cilium: a look at a once-forgotten organelle. Am J Physiol Renal Physiol 2005; 289: F1159-69.

[3] Lin F, Hiesberger T, Cordes K, et al. Kidney-specific inactivation of the KIF3A subunit of kinesin-II inhibits renal ciliogenesis and produces polycystic kidney disease. Proc Natl Acad Sci USA 2003; 100: 5286-91.

[4] Pazour GJ, Dickert BL, Vucica Y, et al. Chlamydomonas IFT88 and its mouse homologue, polycystic kidney disease gene tg737, are required for assembly of cilia and flagella. J Cell Biol 2000; 151: 709-18.

[5] Murcia NS, Richards WG, Yoder BK, et al. The Oak Ridge Polycystic Kidney (orpk) disease gene is required for left-right axis determination. Development 2000; 127: 2347-55.

[6] Yoder BK, Tousson A, Millican L, et al. Polaris, a protein disrupted in orpk mutant mice, is required for assembly of renal cilium. Am J Physiol Renal Physiol 2002; 282: F541-52.

[7] Sharma N, Berbari NF, Yoder BK. Ciliary dysfunction in developmental abnormalities and diseases. Curr Top Dev Biol 2008; 85: 371-427.

[8] Berbari NF, O'Connor AK, Haycraft CJ, Yoder BK. The primary cilium as a complex signaling center. Curr Biol 2009; 19: R526-35.

[9] Molla-Herman A, Boularan C, Ghossoub R, et al. Targeting of beta-arrestin 2 to the centrosome and primary cilium: role in cell proliferation control. PLoS ONE 2008; 3: e3728.

[10] Lingle WL, Salisbury JL. Altered centrosome structure is associated with abnormal mitoses in human breast tumors. Am J Pathol 1999; 155: 1941-51.

[11] Stirling JW, Chandler JA. Ultrastructural studies of the female breast: I. $9+0$ cilia in myoepithelial cells. Anat Rec 1976; 186: 413-6.

[12] Schraml P, Frew IJ, Thoma CR, et al. Sporadic clear cell renal cell carcinoma but not the papillary type is characterized by severely reduced frequency of primary cilia. Mod Pathol 2009; 22: 31-6.

[13] Wong SY, Seol AD, So PL, et al. Primary cilia can both mediate and suppress Hedgehog pathway-dependent tumorigenesis. Nat Med 2009; 15: 1055-61.

[14] Han YG, Kim HJ, Dlugosz AA, et al. Dual and opposing roles of primary cilia in medulloblastoma development. Nat Med 2009; 15: 1062-5.

[15] Gulino A, Arcella A, Giangaspero F. Pathological and molecular heterogeneity of medulloblastoma. Curr Opin Oncol 2008; 20: 66875.

[16] Seeley ES, Carriere C, Goetze T, Longnecker DS, Korc M. Pancreatic cancer and precursor pancreatic intraepithelial neoplasia lesions are devoid of primary cilia. Cancer Res 2009; 69: 422-30.

[17] Nielsen SK, Mollgard K, Clement CA, et al. Characterization of primary cilia and Hedgehog signaling during development of the human pancreas and in human pancreatic duct cancer cell lines. Dev Dyn 2008; 237: 2039-52.

[18] Moser JJ, Fritzler MJ, Rattner JB. Primary ciliogenesis defects are associated with human astrocytoma/glioblastoma cells. BMC Cancer 2009; 9: 448.

[19] Inglis PN, Boroevich KA, Leroux MR. Piecing together a ciliome. Trends Genet 2006; 22: 491-500.

[20] Wood LD, Parsons DW, Jones S, et al. The genomic landscapes of human breast and colorectal cancers. Science 2007; 318: 1108-13.

[21] Sjoblom T, Jones S, Wood LD, et al. The consensus coding sequences of human breast and colorectal cancers. Science 2006; 314: 268-74.

[22] Arts HH, Cremers FP, Knoers NV, Roepman R. Focus on molecules: RPGRIP1. Exper Eye Res 2009; 88: 332-3.

[23] Bartoloni L, Blouin JL, Maiti AK, et al. Axonemal beta heavy chain dynein DNAH9: cDNA sequence, genomic structure, and investigation of its role in primary ciliary dyskinesia. Genomics 2001; 72: 21-33

[24] Hoyer-Fender S. Centriole maturation and transformation to basal body. Semin Cell Dev Biol 2009; 21(2):142-7.

[25] Makino K, Umeda K, Uezu A, et al. Identification and characterization of the novel centrosomal protein centlein. Biochem Biophys Res Commun 2008; 366: 958-62.

[26] Spektor A, Tsang WY, Khoo D, Dynlacht BD. Cep97 and CP110 suppress a cilia assembly program. Cell 2007; 130: 678-90.

[27] Tsang WY, Bossard C, Khanna H, et al. CP110 suppresses primary cilia formation through its interaction with CEP290, a protein deficient in human ciliary disease. Devel Cell 2008; 15: 187-97.

[28] Zhang J, Lipinski RJ, Gipp JJ, Shaw AK, Bushman W. Hedgehog pathway responsiveness correlates with the presence of primary cilia on prostate stromal cells. BMC Dev Biol 2009; 9: 50.

[29] Pugacheva EN, Jablonski SA, Hartman TR, Henske EP, Golemis EA. HEF1-dependent Aurora A activation induces disassembly of the primary cilium. Cell 2007; 129: 1351-63.

[30] Hooper JE, Scott MP. Communicating with Hedgehogs. Nat Rev Mol Cell Biol 2005; 6: 306-17.

[31] Paces-Fessy M, Boucher D, Petit E, Paute-Briand S, BlanchetTournier MF. The negative regulator of Gli, Suppressor of fused (Sufu), interacts with SAP18, Galectin3 and other nuclear proteins. Biochem J 2004; 378: 353-62.

[32] Rohatgi R, Milenkovic L, Scott MP. Patched1 regulates hedgehog signaling at the primary cilium. Science 2007; 317:372-6.

[33] Corbit KC, Aanstad P, Singla V, et al. Vertebrate smoothened functions at the primary cilium. Nature 2005; 437: 1018-21.

[34] Lipinski RJ, Gipp JJ, Zhang J, Doles JD, Bushman W. Unique and complimentary activities of the Gli transcription factors in Hedgehog signaling. Exp Cell Res 2006; 312: 1925-38.

[35] Kasper M, Regl G, Frischauf AM, Aberger F. GLI transcription factors: mediators of oncogenic Hedgehog signalling. Eur J Cancer 2006; 42: 437-45.

[36] Haycraft CJ, Banizs B, Aydin-Son Y, et al. Gli2 and gli3 localize to cilia and require the intraflagellar transport protein polaris for processing and function. PLoS Genet 2005; 1: e53.

[37] Wolf I, Bose S, Desmond JC, et al. Unmasking of epigenetically silenced genes reveals DNA promoter methylation and reduced expression of PTCH in breast cancer. Breast Cancer Res Treat 2007; 105(2):139-55.

[38] Moraes RC, Zhang X, Harrington N, et al. Constitutive activation of smoothened (Smo) in mammary glands of transgenic mice leads to increased proliferation, altered differentiation and ductal dysplasia. Development 2007; 134(6):1231-42.

[39] Mukherjee S FN, Sadlonova A, Novak Z, et al. Hedgehog signaling and response to cyclopamine differs in epithelial and stromal cells in benign breast and breast cancer. Cancer Biol Ther 2006; 5: 67483.

[40] Kubo M, Nakamura M, Tasaki A, et al. Hedgehog signaling pathway is a new therapeutic target for patients with breast cancer. Cancer Res 2004; 64: 6071-4.

[41] Dennler S, Andre J, Alexaki I, et al. Induction of sonic hedgehog mediators by transforming growth factor-beta: Smad3-dependent activation of Gli2 and Gli1 expression in vitro and in vivo. Cancer Res 2007; 67: 6981-6.

[42] Stecca B, Mas C, Clement V, et al. Melanomas require HEDGEHOG-GLI signaling regulated by interactions between GLI1 and the RAS-MEK/AKT pathways. Proc Natl Acad Sci USA 2007; 104: 5895-900.

[43] Schneider L, Clement CA, Teilmann SC, et al. PDGFRalphaalpha signaling is regulated through the primary cilium in fibroblasts. Curr Biol 2005; 15: 1861-6.

[44] Jechlinger M, Sommer A, Moriggl R, et al. Autocrine PDGFR signaling promotes mammary cancer metastasis. J Clin Invest 2006; 116: 1561-70.

[45] Carvalho I, Milanezi F, Martins A, Reis RM, Schmitt F. Overexpression of platelet-derived growth factor receptor alpha in breast cancer is associated with tumour progression. Breast Cancer Res 2005; 7: R788-95.

[46] Schrick JJ, Onuchic LF, Reeders ST, et al. Characterization of the human homologue of the mouse $\mathrm{Tg} 737$ candidate polycystic kidney disease gene. Hum Mol Genet 1995; 4: 559-67.

[47] Praetorius HA, Spring KR. Bending the MDCK cell primary cilium increases intracellular calcium. J Membr Biol 2001; 184: 71-9. 
[48] Praetorius HA, Spring KR. The renal cell primary cilium functions as a flow sensor. Curr Opin Nephrol Hypertens 2003; 12: 517-20.

[49] Hughes J, Ward CJ, Peral B, et al. The polycystic kidney disease 1 (PKD1) gene encodes a novel protein with multiple cell recognition domains. Nat Genet 1995; 10: 151-60.

[50] Mochizuki T, Wu G, Hayashi T, et al. PKD2, a gene for polycystic kidney disease that encodes an integral membrane protein. Science 1996; 272: 1339-42.

[51] Nauli SM, Alenghat FJ, Luo Y, et al. Polycystins 1 and 2 mediate mechanosensation in the primary cilium of kidney cells. Nat Genet 2003; 33: 129-37.

[52] Veeman MT, Axelrod JD, Moon RT. A second canon. Functions and mechanisms of beta-catenin-independent Wnt signaling. Devel Cell 2003; 5: 367-77.

[53] Kikuchi A, Yamamoto H, Kishida S. Multiplicity of the interactions of Wnt proteins and their receptors. Cell Signal 2007; 19: 659-71.

[54] Dabdoub A, Kelley MW. Planar cell polarity and a potential role for a Wnt morphogen gradient in stereociliary bundle orientation in the mammalian inner ear. J Neurobiol 2005; 64: 446-57.

[55] Ross AJ, May-Simera H, Eichers ER, et al. Disruption of BardetBiedl syndrome ciliary proteins perturbs planar cell polarity in vertebrates. Nat Genet 2005; 37: 1135-40.

[56] Park TJ, Haigo SL, Wallingford JB. Ciliogenesis defects in embryos lacking inturned or fuzzy function are associated with failure of planar cell polarity and Hedgehog signaling. Nat Genet 2006; 38: 303-11

[57] Simons M, Gloy J, Ganner A, et al. Inversin, the gene product mutated in nephronophthisis type II, functions as a molecular switch between Wnt signaling pathways. Nat Genet 2005; 37: 53743.

[58] Wu J, Du H, Wang X, et al. Characterization of primary cilia in human airway smooth muscle cells. Chest 2009; 136: 561-70.

[59] Poole CA, Jensen CG, Snyder JA, et al. Confocal analysis of primary cilia structure and colocalization with the Golgi apparatus in chondrocytes and aortic smooth muscle cells. Cell Biol Int 1997; 21: 483-94

[60] Poole CA, Zhang ZJ, Ross JM. The differential distribution of acetylated and detyrosinated alpha-tubulin in the microtubular cytoskeleton and primary cilia of hyaline cartilage chondrocytes. J Anat 2001;199: 393-405.

[61] Jensen CG, Poole CA, McGlashan SR, et al. Ultrastructural, tomographic and confocal imaging of the chondrocyte primary cilium in situ. Cell Biol Int 2004; 28: 101-10.

[62] Housset C. Cystic liver diseases. Genetics and cell biology. Gastroenterol Clin Biol 2005; 29: 861-9.

[63] Gradilone SA, Masyuk AI, Splinter PL, et al. Cholangiocyte cilia express TRPV4 and detect changes in luminal tonicity inducing bicarbonate secretion. Proc Natl Acad Sci USA 2007; 104: 1913843.

[64] Robert A, Margall-Ducos G, Guidotti JE, et al. The intraflagellar transport component IFT88/polaris is a centrosomal protein regulating G1-S transition in non-ciliated cells. J Cell Sci 2007; 120: 628-37.

[65] Yuan K, Frolova N, Xie Y, et al. Primary cilia are decreased in breast cancer: analysis of a collection of human breast cancer cell lines and tissues. J Histochem Cytochem 2010; 58: 857-70.

(C) Yuan et al.; Licensee Bentham Open.

This is an open access article licensed under the terms of the Creative Commons Attribution Non-Commercial License (http://creativecommons.org/licenses/by-nc/ $3.0 /$ ) which permits unrestricted, non-commercial use, distribution and reproduction in any medium, provided the work is properly cited. 